\title{
Event-Driven Acquisition and Machine-Learning-Based Efficient Prediction of the Li-Ion Battery Capacity
}

\author{
Saeed Mian Qaisar ${ }^{1,2}$ [D $\cdot$ Amal Essam EIDin AbdelGawad $^{1} \cdot$ Kathiravan Srinivasan $^{3}$
}

Received: 21 August 2021 / Accepted: 22 September 2021 / Published online: 26 October 2021

(c) The Author(s), under exclusive licence to Springer Nature Singapore Pte Ltd 2021

\begin{abstract}
In almost all contemporary power systems, the battery is an elementary component, and it is routinely used in a variety of critical applications such as drones, avionics, and cell phones. Due to their superior characteristics compared to the concurrent technologies, Li-ion batteries are widely utilized. Since batteries are costly, their usage is closely monitored by battery management systems (BMSs). It ensures that batteries survive and serve longer. Modern BMSs' are complex and sophisticated and can deal with hundreds of cells in a battery pack. It results in an increased processing resources requirement and can cause an overhead power consumption. The aim of this work is to improve current BMSs by redesigning their associative processing chain. It focuses on improving data collection, processing and prediction processes for Li-ion battery cell capacities. To prevent the processing of a large amount of unnecessary data, the classical sensing approach that is fix-rate is avoided and replaced by event-driven sensing (EDS) mechanism to digitize battery cell parameters such as voltages, currents, and temperatures in a way that allows for real-time data compressing. A new approach is proposed for event-driven feature extraction. The robust machine-learning algorithms are employed for processing the extracted features and to predict the capacity of considered battery cell. Results show a considerable compression gain with a correlation coefficient of 0.999 and the relative absolute error (RAE) and root relative squared error (RRSE) of $1.88 \%$ and $2.08 \%$, respectively.
\end{abstract}

Keywords Event-driven processing $\cdot \mathrm{Li}$-ion battery $\cdot$ Remaining useful life $\cdot$ Battery capacity $\cdot$ Features extraction . Computational complexity

\section{Introduction}

The Paris Agreement, which was signed in September 2015, aims to make energy more affordable and sustainable. Its goal is to make cities and human settlements safer and longlasting [1]. After the percentage of greenhouse gases (GHG) emissions in the Earth's atmosphere rose to new record

This article is part of the topical collection "Intelligent Systems" guest edited by Geetha Ganesan, Lalit Garg, Renu Dhir, Vijay Kumar and Manik Sharma.

Saeed Mian Qaisar

sqaisar@effatuniversity.edu.sa

1 College of Engineering, Effat University, Jeddah 22332, Saudi Arabia

2 Communication and Signal Processing Lab, Energy and Technology Research Center, Effat University, Jeddah 22332, Saudi Arabia

3 School of Computer Science and Engineering, Vellore Institute of Technology (VIT), Vellore, India levels in 2019, it became the second warmest year on record and the warmest year in the last decade (2010-2019). In the first quarter of 2020, demand for carbon fuels had recorded its most significant decline, as the climate improved, and global $\mathrm{CO}_{2}$ emissions fell twice as much as the previous total declines since the end of World War II in 1945. Despite that, it was only a temporary improvement in the climate, and once the global economy begins to recover from the Covid-19 pandemic, that escalation in GHG levels will return to the increase $[2,3]$. The planet is making progress in achieving the 7th SDG, as energy is more sustainable and available, renewable energy efficiency has improved, its use expanded, and its economic performance in the electricity sector has improved [4]. However, the fact that these renewable energy sources, such as wind and solar, are intermittent. As a result, they should be combined with energy storage modules. That is why the importance of renewable power storage has increased, as it provides a practical solution for network control and power stability-enhancing [5]. 
Most of today's devices use Li-ion batteries for several reasons, including their lighter weights and higher specific energy density (23-70 Wh/kg) with very high efficiency (close to $90 \%$ ). This type of battery also has a relatively long-life cycle, with an average of 3000 cycles at a discharge of $80 \%$; besides, it does not require a lot of maintenance and has no memory effect. Due to these enhanced attributes, we currently can find the Li-ion batteries within our day-today used electronics [6-10]. Most of all, the high energy and power density of this type of batteries make them compatible with hybrid and electric vehicles (HEVs and EVs), which are the future direction of many companies worldwide. Moreover, its high efficiency makes it usable in various applications, such as improving the quality of renewable energies such as wind and solar energies. Thus, the previous advantages make the Li-ion batteries active contributors to the use of renewable energies on a wide scale [11].

The Li-ion batteries, despite all these advantages, also degrade with time like other rechargeable batteries. The degradation is a kind of aging in which the battery's materials deteriorate over its lifetime, so its performance degrades through operation over time. Every time the battery goes through the charging/discharging cycles, the degradation process accelerates, making the battery's efficiency, power, and capacity further affected. This degradation could lead to unwanted events that could range from inconvenience, financial loss, or fatal tragedies, depending on the application. Therefore, it is necessary to assess and determine a battery's expected lifetime. To avoid the factors that lead to damage to the battery and to extend its life, the BMSs have previously been created and are extensively used [8]. There are some indicators or estimators that represent the monitoring part of the BMS, such as state of charge (SoC); an estimator of the remaining charge capacity, state of health $(\mathrm{SoH})$; a predictor of the discharge capacity that would provide an estimation of the battery age and remaining useful life (RUL); an indicator to the remaining life of a battery until its end of life (EoL). This paper works on the RUL prediction for Li-ion battery cells.

Many academics and industrial practitioners have stepped up their efforts to create new methodologies and techniques for accurately estimating capacities of individual cells in a battery pack. Onward, it can be translated into the remaining useful life (RUL). The particle filter (PF) method, or else known as the sequential Monte Carlo (SMC) method, is a method that importance sampling with Bayesian learning techniques. Qiang et al. [7] proposed an improved version of that method by developing the unscented particle filter (UPF) method, which works by obtaining the proposal distribution by applying the unscented Kalman filter (UKF) algorithm first, then get the final results by applying the PF algorithm. Moreover, several researchers in the literature have used regression models to act as a predictive method for Li-ion batteries. As statistical methods [12], Long et al. [9] proposed an improved autoregressive (AR) mode by utilizing the root mean squared error (RMSE) after stating that a uniform criterion that would provide AR order determination does not exist. The enhanced particle swarm optimization (PSO) algorithm was also applied to determine the best AR model order. Metabolism of the information within the data allowed the AR model order to change adaptively. This method accurately predicts the RUL of Li-ion batteries with a marginal error and it can also be used for onboard applications, according to the researchers [12]. In Ref. [13], authors have tested the reliabilities of rechargeable Li-ion batteries, particularly implanted in medical devices, using a non-linear kernel regression model. The k-nearest neighbor $(\mathrm{kNN})$ regression was used to develop the model. The battery charge voltage and current curves were used in the study. The adoption of the $\mathrm{kNN}$ regression model allows for the incorporation of the user-defined properties of charge curves. The application of PSO reduces the cross-validation (CV) error in the battery cells capacities estimation. Eventually, based on the assessment of 10 years of cycling data, it is determined that the suggested model properly predicted the life of the Li-ion battery cells.

In Ref. [14], the authors have devised another variant of the regression model to estimate the real-time RUL of Li-ion batteries. It is based on the data-driven approaches and employs the machine-learning algorithms. In this context, a mix of the support vector regression (SVR) and support vector machine (SVM) is developed. The goal of the model was to develop an algorithm that might be used in electric vehicles to anticipate when a battery is approaching its end of life (EoL) and alert the driver before it completely depletes. The model was fed with cycling data of batteries under different circumstances extracted from the intended battery dataset. That model has been tested using numerous case studies and has been found to be an effective tool for the RUL estimation of EVs batteries.

\section{Materials and Methods}

The principle of the suggested system is shown in Fig. 1.

\section{Dataset}

The suggested method is tested using the Li-ion battery dataset, provided publicly by the NASA Ames Prognostics Center of Excellence (PCoE) [15]. The considered dataset
Fig. 1 The system's block diagram

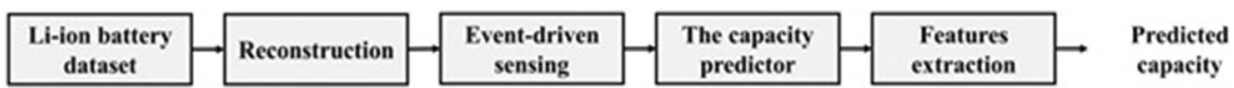


consists of three operating characteristics of high-power Li-ion battery cells: charge, discharge and electrochemical impedance spectroscopy as a function of temperature. The experimentation is carried out at various load currents. The battery cells are drained until the terminal voltage dropped to preset thresholds, which the manufacturer specified should be below $2.7 \mathrm{~V}$. Respecting this threshold avoids the deep discharge aging effects. These batteries were charged and discharged often to accelerate the process of aging. The tests were only terminated after the targeted cells achieved the end of life (EoL) requirement of $30 \%$ decline in rated capacity.

\section{Reconstruction}

To evaluate the event-driven sensing element, the anticipated Li-ion battery parameters waveforms are up-sampled by a factor of one hundred. Upsampling is achieved using an appropriate cascading of the spline interpolators [16]. It provides an analog like representation of the waveforms of the battery parameters, which are utilized as an input to the event-driven sensing stage.

\section{Event-Driven Acquisition}

The sensing and recording of battery cells parameters is an elementary part of modern BMSs. It is based on analogto-digital converter (ADC). Conventional ADCs take samples based on Nyquist's theory and hence does not perform well, while dealing with the irregular and sporadic signals, such as battery cell's voltages and currents $[16,17]$. In this situation, however, Event-Driven ADC (EDADC) is a great option since it adjusts the sampling frequency in response to changes in the incoming signal.

In the case of EDADC, the sampling frequency is adjusted according to changes in the analog signals, $\tilde{x}(t)$. Samples are acquired as a function of the preset thresholds $[17,18]$. The approach is given by the following equation:

$t_{n}=t_{n-1}+d t_{n}$,

where $t_{n}$ is the present sampling instant which is $d t_{n}$ instants later than the previous one, $t_{n-1}$. Likewise, $x_{n}$ is the present sampling amplitude, and $x_{n-1}$ is the previous one. The pair $\left(x_{n}, t_{n}\right)$ is presenting the amplitude-time pair of $n$th sample.

The EDADC only collects the information that is relevant to the situation and discards the rest. As a result, as compared to their classical counterparts, the collected samples exhibit a significant real-time reduction and compression gain. Furthermore, the EDADC lowers pre-processing time and increases system processing and power efficiency [16, $17,19]$.

\section{Feature Extraction}

Battery cell charging/discharging curves for voltages and currents indicate valuable information about battery life. Furthermore, the shape-dependent on the cell's charging/ discharging voltages and currents may be retrieved by working separately on the charging and discharging cycles of the target cell. The intended Li-ion battery cell's actual capacity may be found by combining these features. It enables the RUL to be determined.

Once there is an intersection, an event-driven method with several thresholds is used to extract features. These features are extracted based on shape context-based. It is feasible to derive the shape-dependent content of the cell charging and discharging voltages and currents by separately examining the desired cell's charging and discharging cycles [20]. These mined characteristics may then be utilized to determine the present status of the Li-ion cell in consideration.

When considering discharging cycles, for example, the cell terminal voltage attributes for the $k$ th charging cycle may be derived using the following equation:

$\left(t_{\mathrm{ED} k}, \mathrm{TH}_{b}\right)$, where $I_{\mathrm{DC} k}=\mathrm{TH}_{b}$, and $k=1,2, \ldots, M$,

where $t_{\mathrm{ED} k}$ is the $k$ th instant when the battery terminal voltage reaches the preset threshold, $\mathrm{TH}_{b}, b$ is the threshold index, $I_{\mathrm{DC} k}$ is the $k$ th cell terminal discharging current, and $M$ is the total number of discharging cycles that have been considered.

The attributes from the voltage and current waveforms of each discharging cycle are derived using the same method.

\section{Classification Methods}

Artificial neural network (ANN), k-nearest neighbor (kNN), and random tree (RT) are three robust machine-learning approaches that are used for the prediction of capacity. The mentioned predictors have long been considered the most commonly used machine-learning paradigms and have proven capable of learning and predicting more complex data patterns in many application systems.

\section{- Artificial neural network (ANN)}

The ANN is one of the most robust and effective algorithms for predicting complex data, which linear classification algorithms cannot succeed. The general architecture of any ANN consists of neurons representing processing units on three layers, namely the input layer, the hidden layer, and the output layer, where the hidden layer is employed to proceed with the non-linear classification. Adjusting the harmony between those three layers 
determines overall network performance. The more hidden layers are, the lower the error rate [21].

- $\mathrm{k}-\mathrm{Nearest}$ neighbor $(\mathrm{kNN})$

The k-nearest neighbor $(\mathrm{kNN})$ method is a similaritybased technique that is quick and efficient. It creates a non-linear kernel regression model and forecasts the regression of a test element's answer by averaging the responses of k's nearest neighbors to that element [13, 22].

- Random tree (RT)

The RT is one of the best ensemble algorithms. Its working principle is that a robust learner can be built by uniting many weak, randomly formed learners. This algorithm creates many decision branches at different nodes; each produces a random classification of the data sample input; the RT collects these decisions and gives the output which had the highest vote decision [23].

\section{Performance Evaluation Metrics}

The compression ratio and accuracy of prediction are used to assess the suggested model's performance.

- Compression ratio $\left(R_{\text {Comp }}\right)$

It is given by the following equation:

$R_{\mathrm{Comp}}=\frac{N}{N_{\mathrm{ED}}}$,

where $N$ denotes the number of samples acquired in the classical counterpart, and $N_{\mathrm{ED}}$ denotes the number of samples acquired in the suggested model.

- Accuracy of prediction

The correlation coefficient $(r)$, relative absolute error (RAE), and root relative squared error (RRSE) are used to calculate the accuracy of the Li-ion battery capacity perdition.

The correlation coefficient $(r)$ measures the degree of the relationship between two sets of data, such as present and projected capacities. It takes values between -1 and 1. The uncorrected series is represented by 0 . Whereas the inverse and direct relations are respectively represented by -1 and 1 [24]. The following equation can be used to determine the correlation coefficient, $r$ :

$r=\frac{\sum_{i=1}^{n}\left(c_{i}^{\mathrm{act}}-\overline{c^{\mathrm{act}}}\right)\left(c_{i}^{\mathrm{pred}}-\overline{c^{\mathrm{pred}}}\right)}{\sqrt{\sum_{i=1}^{n}\left(c_{i}^{\mathrm{act}}-\overline{c^{\mathrm{act}}}\right)^{2} \sum_{i=1}^{n}\left(c_{i}^{\mathrm{pred}}-\overline{c^{\mathrm{pred}}}\right)^{2}}}$,

where $c_{i}^{\text {act }}$ denotes the actual capacity for the $i$ th instant, $c_{i}^{\text {pred }}$ denotes the anticipated capacity, $\overline{c^{\text {act }}}$ and $\overline{c^{\text {pred }}}$, respectively, denote the mean of the two, and $n$ is the total number of instants.
The relative absolute error (RAE) and root relative squared error (RRSE) are given by Eqs. (5) and (6), respectively.

Both the RAE and RRSE range from 0 to 1 . The better the prediction, the closer their values are to 0 . Their calculation includes division by the $c_{i}^{\text {act }}$ variation; thus, they are called 'relative' errors. Hence, they are useful in comparing models in terms of accuracy even if their errors are not in similar units, unlike the mean absolute error (MAE) and root mean squared error (RMSE) which are not applicable if the error units are different [25].

$$
\begin{gathered}
\mathrm{RAE}=\frac{\sum_{i=1}^{n}\left|c_{i}^{\mathrm{act}}-c_{i}^{\mathrm{pred}}\right|}{\sum_{i=1}^{n}\left|c_{i}^{\mathrm{act}}-\overline{c^{\mathrm{act}}}\right|}, \\
\mathrm{RRSE}=\sqrt{\frac{\sum_{i=1}^{n}\left(c_{i}^{\mathrm{act}}-c_{i}^{\mathrm{pred}}\right)^{2}}{\sum_{i=1}^{n}\left(c_{i}^{\mathrm{act}}-\overline{c^{\mathrm{act}}}\right)^{2}}} .
\end{gathered}
$$

\section{Results and Discussion}

Shape context-based characteristics of the cell discharging voltages, currents, and temperatures are collected during several discharging cycles using the dataset of cell-5. A total of 168 discharging cycles are taken into account. Each cycle is treated as an instance, with discharge voltage, current, and temperature curves corresponding to it. A MATLAB-based event-driven model is used to extract features. Figure 2 demonstrates the idea. It displays a zoom of the discharging current curves of the considered cell for the discharging cycles 31, 71, 101, and 152 .

As previously stated, conventional sensing generates a large amount of useless data, preventing real-time data reduction. Each instance in the case study is made up of three $3500 \mathrm{~s}$ waveforms. As a result, each instance is made up of 10,500 samples collected at a constant rate of $10 \mathrm{~Hz}$ [15]. The recommended method, on the other hand, only obtains 96 characteristics each instance. The compression ratio is computed, and it shows that the proposed approach has a 109.4-fold average compression increase. This will very certainly result in a considerable reduction in computing complexity and energy usage [26-28].

To test the correctness of the recommended solution, the pre-mentioned performance assessment metrics are computed for each predictor. A ratio of 70:30\% is applied to split the data for training and testing purposes, respectively. Table 1 presents the results summary. 
Fig. 2 Examples of the cell discharging current features extraction, for the discharging cycle numbers $31,71,101$, and 152

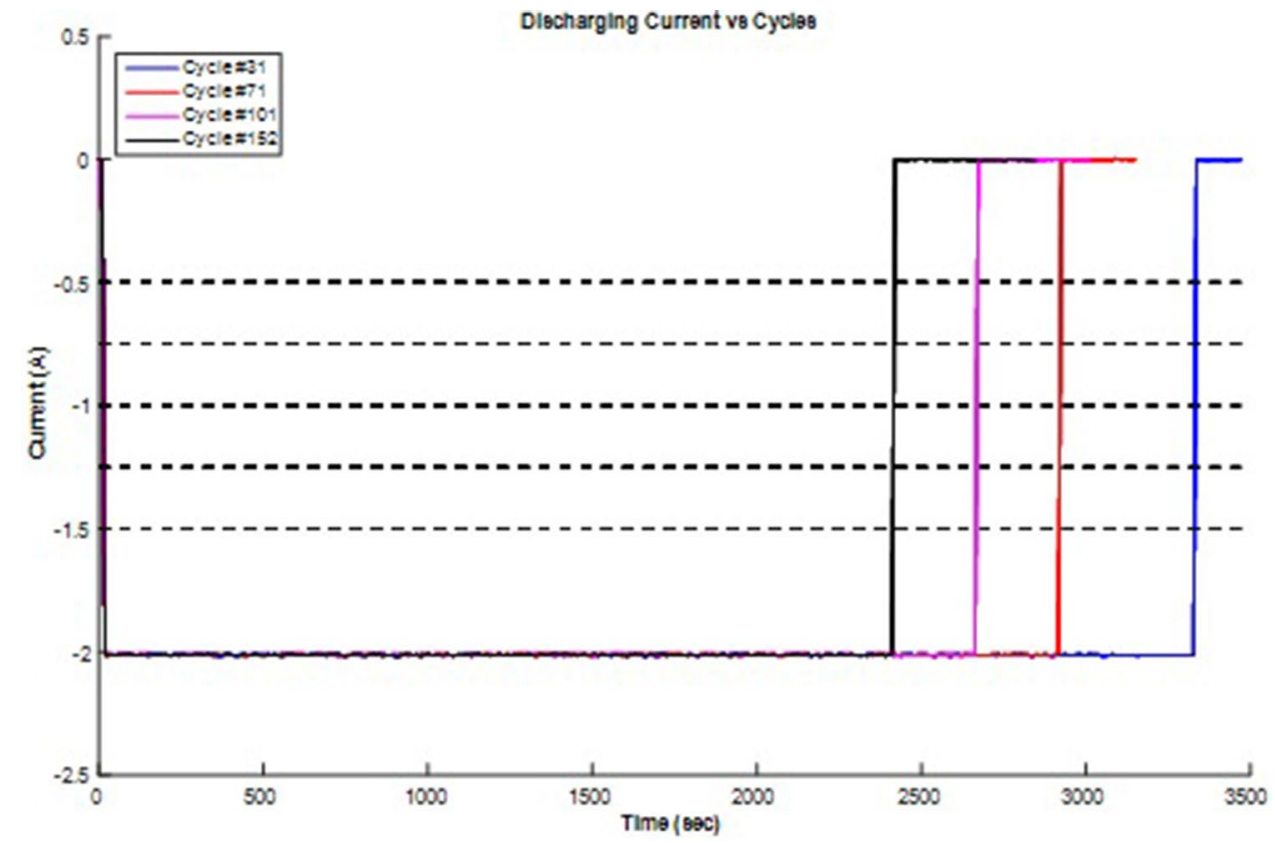

Table 1 Performance evaluation metrics comparison

\begin{tabular}{llll}
\hline Algorithm & $r$ & RAE (\%) & RRSE (\%) \\
\hline ANN & 0.98 & 1.9 & 2.2 \\
kNN & 0.97 & 2.3 & 2.5 \\
RT & 0.95 & 3.3 & 3.4 \\
\hline
\end{tabular}

Table 1 indicates that the ANN achieves superior performance with the maximum correlation coefficient and minimum RAE and RRSE of $0.98,1.9 \%$, and $2.2 \%$, respectively. Among the machine-learning algorithms studied, the $\mathrm{kNN}$ has the second-best performance, while the RT has the lastbest performance. It demonstrates that, in the scenario under consideration, the RT can be easily biased and it can result in its diminished performance as compared to the $\mathrm{kNN}$ and ANN algorithms.

\section{Conclusion}

Due to the high cost of Li-ion batteries, their use is closely monitored by BMSs to optimize their performance and extend their life. Having a Li-ion battery pack monitoring system in the smart grid will help to ensure that the batteries are in a proper state and fit for the various applications. That will contribute to decreasing the risk of failure of Li-ion batteries that leads to vast blackouts.

For determining the capacity cells in a Li-ion battery pack via event-driven features extraction. As a result, it implicitly determines the Li-ion battery's remaining useful life (RUL) to perform real-time data compression. To forecast the capacity of a given cell, robust machine-learning techniques are used.

The results show superior performance for artificial neural network among its studied counterparts. It produces a significant compression gain of 109.4 times on average, with a minimum relative absolute error of $1.8819 \%$ and a root relative squared error of $2.0849 \%$, respectively. Future study will include expanding the work by including the ensemble and deep-learning-based predictors. Another possibility is a real-time embedded realization of the suggested approach.

Funding This project is funded by Effat University, Jeddah, KSA, under the Grant number UC\#9/29 April.2020/7.1-22 (2)4.

\section{Declarations}

Conflict of interest The authors declare that they have no conflict of interest.

\section{References}

1. A global compact for sustainable development - business and the SDGs: acting responsibly and finding opportunities I UN Global Compact. UN Global Compact, 2015. https://www.unglobalco mpact.org/library/2291. Accessed 24 Jan 2021.

2. Global energy and $\mathrm{CO}_{2}$ emissions in 2020 - global energy review 2020 - analysis. IEA, 2020. https://www.iea.org/reports/globalenergy-review-2020/global-energy-and-co2-emissions-in-2020. Accessed 24 Jan 2021.

3. Martin, "Climate Change," United Nations Sustainable Development, 2019. https://www.un.org/sustainabledevelopment/clima te-change/. Accessed 23 Jan 2021. 
4. Martin, "Energy," United Nations Sustainable Development. https://www.un.org/sustainabledevelopment/energy/. Accessed 24 Jan 2021.

5. Alattar AH, et al. Performance enhancement of micro grid system with SMES storage system based on mine blast optimization algorithm. Energies. 2019. https://doi.org/10.3390/en12163110.

6. Xu D, Wang L, Yang J. Research on Li-ion battery management system. In: 2010 International conference on electrical and control engineering, Jun 2010, pp. 4106-9. https://doi.org/10.1109/ iCECE.2010.998.

7. Miao Q, Xie L, Cui H, Liang W, Pecht M. Remaining useful life prediction of lithium-ion battery with unscented particle filter technique. Microelectron Reliab. 2013;53(6):805-10. https://doi. org/10.1016/j.microrel.2012.12.004.

8. Berecibar M, Gandiaga I, Villarreal I, Omar N, Van Mierlo J, Van den Bossche P. Critical review of state of health estimation methods of Li-ion batteries for real applications. Renew Sustain Energy Rev. 2016;56:572-87. https://doi.org/10.1016/j.rser.2015. 11.042 .

9. Long B, Xian W, Jiang L, Liu Z. An improved autoregressive model by particle swarm optimization for prognostics of lithiumion batteries. Microelectron Reliab. 2013;53(6):821-31. https:// doi.org/10.1016/j.microrel.2013.01.006.

10. Zhang H, Miao Q, Zhang X, Liu Z. An improved unscented particle filter approach for lithium-ion battery remaining useful life prediction. Microelectron Reliab. 2018;81:288-98. https://doi.org/ 10.1016/j.microrel.2017.12.036.

11. Nitta N, Wu F, Lee JT, Yushin G. Li-ion battery materials: present and future. Mater Today. 2015;18(5):252-64. https://doi.org/10. 1016/j.mattod.2014.10.040.

12. Sikorska JZ, Hodkiewicz M, Ma L. Prognostic modelling options for remaining useful life estimation by industry. Mech Syst Signal Process. 2011;25(5):1803-36. https://doi.org/10.1016/j.ymssp. 2010.11.018

13. Hu C, Jain G, Zhang P, Schmidt C, Gomadam P, Gorka T. Datadriven method based on particle swarm optimization and k-nearest neighbor regression for estimating capacity of lithium-ion battery. Appl Energy. 2014;129:49-55. https://doi.org/10.1016/j.apenergy. 2014.04.077.

14. Patil MA, et al. A novel multistage support vector machine based approach for Li ion battery remaining useful life estimation. Appl Energy. 2015;159:285-97. https://doi.org/10.1016/j.apenergy. 2015.08.119.

15. Lucu M, Martinez-Laserna E, Gandiaga I, Camblong H. A critical review on self-adaptive Li-ion battery ageing models. J Power Sources. 2018;401:85-101. https://doi.org/10.1016/j.jpowsour. 2018.08.064

16. Qaisar SM, Hussain SF. Effective epileptic seizure detection by using level-crossing EEG sampling sub-bands statistical features selection and machine learning for mobile healthcare. Comput Methods Programs Biomed. 2021;203:106034. https://doi.org/10. 1016/j.cmpb.2021.106034.

17. Qaisar SM, Fesquet L, Renaudin M. Effective resolution of an adaptive rate ADC. 2009. Special-session.

18. Mina Qaisar S, Sidiya D, Akbar M, Subasi A. An event-driven multiple objects surveillance system. Int J Electr Comput Eng Syst. 2018;9(1):35-44.

19. Qaisar SM. Efficient mobile systems based on adaptive rate signal processing. Comput Electr Eng. 2019;79:106462.

20. Ren L, Zhao L, Hong S, Zhao S, Wang H, Zhang L. Remaining useful life prediction for lithium-ion battery: a deep learning approach. IEEE Access. 2018;6:50587-98.

21. Hallinan JS. Chapter 1 - computational intelligence in the design of synthetic microbial genetic systems. In: Harwood C, Wipat A, editors. Methods in microbiology, vol. 40. Cambridge: Academic Press; 2013. p. 1-37. https://doi.org/10.1016/B978-0-12-4170292.00001-7.

22. Fu K-S. Applications of pattern recognition. Boca Raton, Florida, US: CRC Press; 2019.

23. Zhao Y, Zhang Y. Comparison of decision tree methods for finding active objects. Adv Space Res. 2008;41(12):1955-9. https:// doi.org/10.1016/j.asr.2007.07.020.

24. Mun J. Chapter 3 - a primer on quantitative risk analysis. In: Glantz M, Kissell R, editors. Multi-asset risk modeling. San Diego: Academic Press; 2014. p. 63-118. https://doi.org/10.1016/ B978-0-12-401690-3.00003-2.

25. Zavarella L. how to better evaluate the goodness-of-fit of regressions. Medium, Feb. 05, 2019. https://medium.com/microsofta zure/how-to-better-evaluate-the-goodness-of-fit-of-regressions990dbf1c0091. Accessed 24 Feb 2021.

26. Mian Qaisar S. Event-driven coulomb counting for effective online approximation of Li-ion battery state of charge. Energies. 2020;13(21):5600.

27. Qaisar SM. Baseline wander and power-line interference elimination of ECG signals using efficient signal-piloted filtering. Healthc Technol Lett. 2020;7(4):114-8.

28. Mian Qaisar S, Fawad Hussain S. Arrhythmia diagnosis by using level-crossing ECG sampling and sub-bands features extraction for mobile healthcare. Sensors. 2020;20(8):2252.

Publisher's Note Springer Nature remains neutral with regard to jurisdictional claims in published maps and institutional affiliations. 